changes(Figure 1B). MRI hip showed minimal effusion, synovial thickening edema with STIR hyperintensity and enlargement of bilateral femoral epiphysis. MRI knee showed minimal effusion, marrow edema in patella(Figure 1C, arrow head), femoral condyle, diffuse synovial thickening with contrast enhancement(Figure 1D, arrow) and deformed patellar contour. Immunological tests showed negative RF, ACPA and positive ANA(Hep2) speckled 4+. Immunoblot for ENA was negative. His ophthalmological evaluation showed no evidence of uveitis.

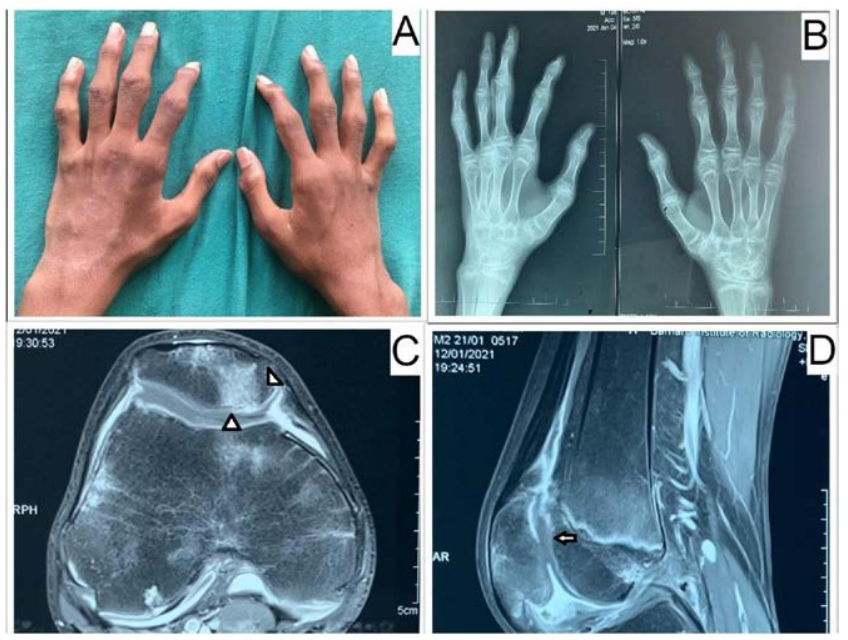

Figure 1. Clinical picture showing typical hand deformity and swelling at bone ends $(A)$, hand radiograph showing epiphyseal enlargement(B), MRI knee T2 STIR showing bone marrow edema(arrow head) in patella(C) and synovial thickening(arrow) with contrast enhancement in fat saturated T1 MRI with contrast.

Results: Patient tested positive for homozygous mutation in WISP-3 gene. He was treated with ibuprofen and supportive measures. Orthopedic consultation obtained and planned for hip, knee replacement during follow up. Follow up imaging and acute phase response was advised after three months.

Conclusion: Although PPRD was classically described as a degenerative disease, the findings presented in our case show coexisting inflammation. Bone marrow edema in weight bearing areas, synovial effusion may be explained as part of cartilage degeneration like in osteoarthritis but synovial hypertrophy with contrast enhancement, power Doppler signals in ultrasound, ANA positivity may be signs of coexisting inflammatory or autoimmune phenomenon.

REFERENCES:

[1] Hurvitz JR, Suwairi WM et al. Mutations in the CCN gene family member WISP3 cause progressive pseudorheumatoid dysplasia. Nat Genet. 1999 Sep;23(1):94-8. doi: 10.1038/12699. PMID: 10471507.

[2] Garcia Segarra N, Mittaz L et al. The diagnostic challenge of progressive pseudorheumatoid dysplasia (PPRD): a review of clinical features, radiographic features, and WISP3 mutations in 63 affected individuals. Am J Med Genet C Semin Med Genet. 2012 Aug 15;160C(3):217-29. doi: 10.1002/ ajmg.c.31333. Epub 2012 Jul 12. PMID: 22791401.

Disclosure of Interests: None declared

DOI: 10.1136/annrheumdis-2021-eular.3480

\section{AB0780 GENDER FEATURES OF VERIFICATION OF DIAGNOSIS UNDIFFERENTIATED ARTHRITIS}

O. Malyshenko ${ }^{1}$, M. Koroleva ${ }^{1}$, M. Letaeva ${ }^{1}$, J. Averkieva ${ }^{1}$, T. Raskina ${ }^{1}$ ${ }^{1}$ Kemerovo State Medical University, Department of Propedeutics of Internal Medicine, Kemerovo, Russian Federation

Background: Undifferentiated arthritis (UDA) is an inflammatory arthritis that does not meet the criteria for any rheumatologic disease. Early verification of UDA is currently one of the main goals of modern rheumatology, since a diagnosis established at an early date allows determining a therapeutic strategy. The high social significance of arthritis lies in the predominant lesion of people of working age, the steady progression of the disease, early disability and a reduction in life expectancy. Objectives: To study the gender characteristics of verification of the diagnosis of undifferentiated arthritis.

Methods: A retrospective analysis of 74 case histories of patients diagnosed with UDA was carried out. The study group consisted of 26 men and 48 women, mean age $50.6 \pm 4.3$ years. All patients underwent a comprehensive laboratory and instrumental examination according to the standard of an articular syndrome of unclear genesis.

Results: According to the data obtained, the duration of the articular syndrome averaged $2.53 \pm 1.2$ years. In 29 patients $(21.6 \%$ of women and $17.6 \%$ of men), on average, after $1.72 \pm 0.9$ years, the diagnosis of NDA was clarified. Taking into account modern diagnostic criteria, the following diseases were verified: rheumatoid arthritis in $13.5 \%$ (12.2\% in women and $1.3 \%$ in men), ankylosing spondylitis in $10.8 \%$ (2.7\% in women and $8.1 \%$ in men). Osteoarthritis, psoriatic arthritis and APS were diagnosed in $5.4 \%, 1.4 \%$ and $1.4 \%$ of women, and gouty arthritis bone tuberculosis and HIV in $4.1 \%, 1.4 \%$ and $1.4 \%$ of men respectively. In $60.8 \%$ (43.2\% in women and $17.6 \%$ in men), the etiology of arthritis was not verified. Conclusion: In a third of patients with UDA, diagnosis verification takes about 2 years on average. In more than half of patients, the diagnosis remains the same. According to the data obtained, rheumatoid arthritis was more often verified in women, while ankylosing spondylitis in men, which is consistent with statistical data. Disclosure of Interests: None declared

DOI: 10.1136/annrheumdis-2021-eular.3507

\section{ONE, NONE, MANY: REFLECTIONS ON A RARE CASE}

G. Sandri ${ }^{1}$, L. Belletti ${ }^{1}$, M. Cavedoni ${ }^{2}$, C. Galluzzo ${ }^{1}$, S. Bruni ${ }^{3}$, M. T. Mascia ${ }^{1}$ ${ }^{1}$ Rheumatology Institute, Department of Clinic and Medical Specialties, Modena, Italy; ${ }^{2}$ General Practitioner, Department of General Medicine, Modena, Italy; ${ }^{3}$ Genzyme, Orchard Therapeutics, Modena, Italy

Background: Rare diseases are all those diseases that present, in the European Union, a prevalence of less than 5 cases per 10,000 people. The number of rare diseases is estimated at roughly 7,000 but there are also longstanding medical conditions that elude diagnosis and could be identified as rare.

Objectives: Demonstrate the importance of international research in orphan diseases.

Methods: We report a case of $44 \mathrm{y} / \mathrm{o}$ female patient who arrived to our observation in 2006. Short stature, early puberty, ligament laxity, BMI <17. From the age of 29 : recurrent diarrhea, pain in the spine, osteolytic lesions in spine and endosteal thickening in long bones, muscle contractures, strength deficit, muscular hypotrophy and hypotonia, cardiac conduction and blood pressure disorders, demyelinating MS-lesions, hyperprolactinaemia, slow wound healing, sicca syndrome, osteoporosis. No familiarity for bone lesions. In 2007 her first son (21y/o) began to complain pain at limbs. The young man presented the same bone lesions as the mother and shortening of the PR, prolactinoma, recurrent diarrhea, short stature, early puberty. Over the years numerous pathologies have been first hypothesized and then excluded: multiple sclerosis, bone metastases, Paget's disease, celiac disease, McCune Albright, Camurati-Engelmann syndrome, mitochondrial disease. No conclusive diagnosis despite the thousands of kilometers traveled, the numerous experts heard and the countless examinations carried out by the patients.

Results: In September 2009, the patients had been investigated at the $\mathrm{NIH}$ (Washington D.C.) during the "Undiagnosed Diseases Program" but without results until 2013 when the patients were informed of the detection of an ATP6V1H gene mutation never described before in humans. The gene encodes a vacuolar ATPase, a multimeric enzyme that plays several roles: is involved in endocytosis, intracellular trafficking, and protein degradation and energy production, appears to be a risk factor in the development of dyslipidemias and type II diabetes, has a bone resorption function. Also in the patient's father were founded the same mutation and asymptomatic bone lesions. In 2016 and 2017 studies have reported mouse models of osteoporosis that were generated by knocking out the ATP6V1H gene.

Conclusion: from this case it is possible to understand the difficulty of diagnosing a rare disease, the need of an international collaboration in research From these studies it can be deduced moreover that the ATP6V1H gene could be an important target for therapeutic interventions aimed at preventing bone resorption and treating osteoporosis; evidence to support exploration of MMP9 and MMP13 as therapeutic targets for patients with ATP6V1H deficiency.This mutation seems to affect only one family, but it is possible that the penetrance of the disease-causing mutation is variable. In literature is reported an enhanced expression of MMP-9 in a variety of autoimmune diseases and neurologica pathologies (2) therefore the mutation can be at the basis of other much more common pathologies.

\section{REFERENCES:}

[1] Zhang $Y$, Huang $H$, Zhao G, Yokoyama T, Vega $H$, Huang $Y$, Sood R, Bishop K, Maduro V, Accardi J, Toro C, Boerkoel CF, Lyons K, Gahl WA, Duan X, Malicdan MC, Lin S. ATP6V1H Deficiency Impairs Bone Development through Activation of MMP9 and MMP13. PLoS Genet. 2017 Feb 3;13(2):e1006481. doi: 10.1371/journal.pgen.1006481.

[2] Ram M, Sherer Y, Shoenfeld Y. Matrix metalloproteinase-9 and autoimmune diseases. J Clin Immunol. 2006 Jul;26(4):299-307. doi: 10.1007/ s10875-006-9022-6.

Disclosure of Interests: Gilda Sandri: None declared, Lorenza Belletti: None declared, Michele Cavedoni: None declared, Claudio Galluzzo: None declared, stefano bruni Consultant of: Genzyme, Employee of: Genzyme, Maria Teresa Mascia: None declared

DOI: 10.1136/annrheumdis-2021-eular.3573 